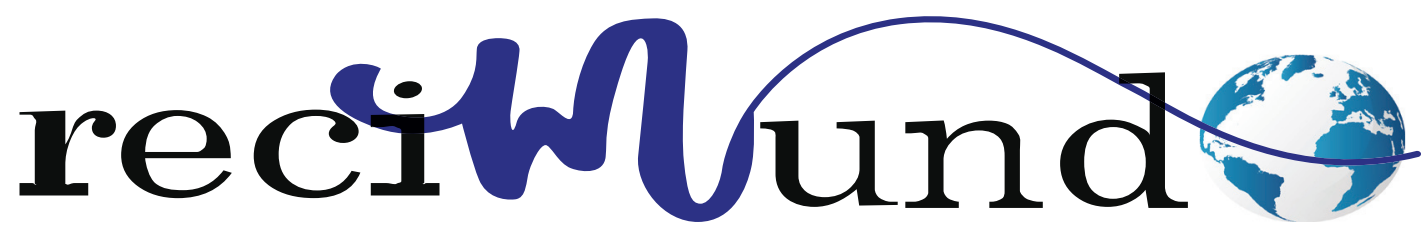

Revista Científica Mundo de la Investigación y el Conocimiento

DOI: 10.26820/recimundo/4.(4).octubre.2020.123-133

URL: http://recimundo.com/index.php/es/article/view/886

EDITORIAL: Saberes del Conocimiento

REVISTA: RECIMUNDO

ISSN: 2588-073X

TIPO DE INVESTIGACIÓN: Artículo de Revisión

CóDIGo UNESCO: 32 Ciencias Médicas; 3201 Ciencias Clínicas

PAGINAS: $123-133$

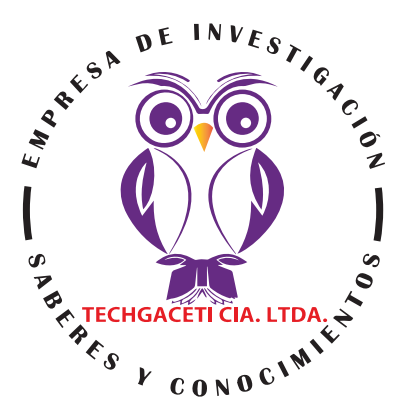

\title{
Escala de Alvarado en apendicitis aguda
}

Alvarado scale in acute appendicitis

Escala de Alvarado em apendicite aguda

Isaac Fernando Díaz Castro'; Castillo Olvera Jonathan Alberto2;

Susan Michelle Erazo Haro ${ }^{3}$; Franklin Iván Moreno Castro ${ }^{4}$

RECIBIDO: 10/07/2020 ACEPTADO: 26/08/2020 PUBLICADO: 15/10/2020

1. Médico; Hospital Naval de Guayaquil; Guayaquil, Ecuador; isaacfernandodiazcastro@gmail.com; (iD https://orcid.org/00000003-2727-2105

2. Médico; Área de Emergencias; Hospital Naval de Guayaquil; Guayaquil, Ecuador; jonathan_athlerm@hotmail.com; https://orcid.org/0000-0002-1089-9297

3. Médico; Investigadora Independiente; Guayaquil, Ecuador; suerazo18@gmail.com; iD https://orcid.org/0000-0002-90750029

4. Médico; Investigador Independiente; Guayaquil, Ecuador; frankfimcmoreno@gmail.com (iD https://orcid.org/0000-00024363-2775

\section{CORRESPONDENCIA}

Isaac Fernando Díaz Castro

isaacfernandodiazcastro@gmail.com

Guayaquil, Ecuador 


\section{RESUMEN}

La Apendicitis Aguda (AA) se trata de un proceso inflamatorio del apéndice cecal (o vermiforme), frecuentemente a causa de la obstrucción del lumen del apéndice. También se asocia con un riesgo elevado de desarrollar íleo, peritonitis, absceso abdominal sino tiene un tratamiento rápido y oportuno. Las herramientas tecnológicas como la TC, la ecografía y la IRM constituyen en la actualidad el complemento idóneo para el diagnóstico de la AA, que inicialmente se basa en la clínica, no obstante, esta posibilidad aún no se da en la mayoría de los centros hospitalarios y servicios de urgencia a nivel mundial, de allí la trascendencia otros métodos diagnósticos en este tipo de patologías, como resulta ser el caso de la Escala de Alvarado. Es precisamente en base a ese tipo de situaciones que surgió el interés por desarrollar el presente estudio, el cual tuvo por objeto explorar e identificar, mediante el uso de bases de datos y buscadores especializados, fuentes vigentes con las cuales referir y sustentar en qué consiste el uso de la Escala de Alvarado para el diagnóstico de la AA, razón por la que se decidió efectuar una investigación de diseño bibliográfico, bajo una metodología de revisión. Con los resultados se deja evidencia de las distintas fuentes y recursos encontrados, mismos que fundamentan algunas acepciones de la AA, etiología, síntomas y diagnosis, siendo en éste último el punto en el que se hace énfasis respecto a la Escala de Alvarado, aludiendo a los resultados obtenidos en otros estudios. En definitiva, la Escala de Alvarado reduce el tiempo de evolución preoperatorio al acelerar la identificación del cuadro clínico, aspecto por demás trascendental ante situaciones de centros de atención de la salud y/o servicios de emergencia con condiciones deficientes o inexistentes de equipamiento imagenológico apropiado para este tipo de patologías.

Palabras clave: Enfermedad intrabdominal, abdomen agudo quirúrgico, sensibilidad, especificidad, apendicetomía.

\section{ABSTRACT}

Acute Appendicitis (AA) is an inflammatory process of the cecal (or vermiform) appendix, frequently due to the obstruction of the lumen of the appendix. It is also associated with a high risk of developing ileus, peritonitis, abdominal abscess if it does not have a fast and timely treatment. Technological tools such as CT, ultrasound and MRI are currently the ideal complement for the diagnosis of AA, which is initially based on the clinic, however, this possibility still does not exist in most hospitals and emergency services worldwide, hence the importance of other diagnostic methods in this type of pathology, as is the case with the Alvarado Scale. It is precisely on the basis of these types of situations that the interest arose to develop this study, which aimed to explore and identify, through the use of databases and specialized search engines, current sources with which to refer and support what it consists of. the use of the Alvarado Scale for the diagnosis of AA, which is why it was decided to carry out a bibliographic design research, under a review methodology. With the results, evidence is left of the different sources and resources found, which support some meanings of AA, etiology, symptoms and diagnosis, the latter being the point in which emphasis is made regarding the Alvarado Scale, alluding to the results obtained in other studies. In short, the Alvarado Scale reduces the preoperative evolution time by accelerating the identification of the clinical picture, an aspect that is transcendental in situations of health care centers and / or emergency services with deficient or non-existent conditions of appropriate imaging equipment for this type of pathologies.

Keywords: Intra-abdominal disease, acute surgical abdomen, sensitivity, specificity, appendectomy.

\section{RESUMO}

A apendicite aguda (AA) é um processo inflamatório do apêndice cecal (ou vermiforme), freqüentemente devido à obstrução da luz do apêndice. Também está associada a um alto risco de desenvolver íleo, peritonite, abscesso abdominal se não houver um tratamento rápido e oportuno. Ferramentas tecnológicas como tomografia, ultrassom e ressonância magnética são atualmente o complemento ideal para o diagnóstico de AA, que é inicialmente baseado na clínica, porém, essa possibilidade ainda não existe na maioria dos hospitais e serviços de emergência em todo o mundo, daí a importância de outros diagnósticos métodos nesse tipo de patologia, como é o caso da Escala de Alvarado. É precisamente com base neste tipo de situações que surge o interesse em desenvolver este estudo, que teve como objetivo explorar e identificar, através da utilização de bases de dados e motores de busca especializados, as fontes atuais para referenciar e apoiar o seu conteúdo. a utilização da Escala de Alvarado para o diagnóstico de AA, razão pela qual se optou por realizar uma pesquisa de delineamento bibliográfico, sob metodologia de revisão. Com os resultados, ficam evidências das diferentes fontes e recursos encontrados, que sustentam alguns significados de AA, etiologia, sintomas e diagnóstico, sendo este último o ponto em que se dá ênfase à Escala de Alvarado, aludindo aos resultados obtidos em outras estudos. Em suma, a Escala de Alvarado reduz o tempo de evolução pré-operatória por acelerar a identificação do quadro clínico, aspecto que é transcendental em situações de centros de saúde e / ou serviços de emergência com condições deficientes ou inexistentes de equipamentos de imagem adequados para este tipo de patologias.

Palavras-chave: Doença intra-abdominal, abdome cirúrgico agudo, sensibilidade, especificidade, apendicectomia. 


\section{Introducción}

Conforme al criterio aportado por el Grupo BMJ (British Medical Journal) expuesto mediante el desarrollo de BMJ Best Practice (Mejores Prácticas BMJ) (2020); herramienta de contenido que ésta división proporciona en base a la constante recaudación de evidencia de investigación actualizada y la opinión de expertos internacionales, ha de saberse que, entre las emergencias abdominales quirúrgicas agudas más comunes se encuentra la apendicitis aguda (AA). De la misma manera se indica que, a lo largo de la vida, generalmente el $8.6 \%$ de los hombres y el $6.7 \%$ de las mujeres corren el riesgo de desarrollar una apendicitis aguda.

A nivel mundial, la incidencia conjunta de apendicitis o apendicetomía es de alrededor de 100 por cada 100,000 habitantes. Los datos sugieren un rápido aumento de la incidencia en los países recientemente industrializados. La afección se observa con mayor frecuencia en pacientes de edades comprendidas entre los primeros años de la adolescencia y los últimos años de la década de los 40. Hay un ligero predominio masculino sobre femenino (1.3:1). (BMJ Best Practice, 2020, pág. 4)

Meléndez, Cosio, \& William (2019) también han fundamentado que "en muchos países latinoamericanos la apendicitis aguda es la principal causa de procedimientos quirúrgicos de la región abdominal" (pág. 14). De igual forma indicaron que la AA se identifica por la presencia de dolor abdominal que se desplaza hacia la fosa iliaca derecha, dolor en el cuadrante inferior derecho, dolor al rebote o descompresión, fiebre, anorexia, náuseas y, en ocasiones, vómito.

La sospecha y diagnóstico de apendicitis aguda se basa, predominantemente, en la clínica. En muchos casos, principalmente durante la fase prodró- mica, las manifestaciones clínicas pueden ser vagas e inciertas e, incluso, similares a otras afecciones. La falla en reconocer tempranamente el diagnóstico eleva la mortalidad y la morbilidad de la enfermedad y el riesgo de complicaciones. El retraso en el diagnóstico incrementa costos en el servicio de urgencias y hospitalario. (Motta et al., 2017, pág. 322)

El diagnóstico de AA es posible mediante varios métodos, y más recientemente destacan la tomografía computarizada (TC), la ecografía y la imagen por resonancia magnética (IRM), estas últimas son más propias en casos de mujeres embarazadas (BMJ Best Practice, 2020), pero; según Fernández, Sarango, Lema, \& Ibarra (2019), estas herramientas tecnológicas (que facilitarían una diagnosis más oportuno y certera) no siempre están disponibles para el personal capacitado en los centros asistenciales de salud; y, a parte de otras particularidades que dificultan el diagnóstico asociadas al paciente (sobrepeso, aumento de umbral del dolor) entre otras, es que entonces la Escala (o test) de Alvarado representa una valiosa solución diagnostica de la AA.

La Escala de Alvarado fue una propuesta que en 1986 por el médico colombiano Alfredo Alvarado, tras los resultados obtenidos mediante un estudio retrospectivo con 305 pacientes intervenidos quirúrgicamente por apendicitis aguda y de los que, según Motta et al. (2017) "tabuló los síntomas y signos de la enfermedad al igual que los resultados de laboratorio de los enfermos" (pág. 327). Con dicho trabajo desarrolló una escala que determina 8 parámetros 0 características clínicas predictivas, agrupadas bajo el acrónimo MANTRELS (por sus siglas en inglés) asignándoles a seis de esos el valor de 1 punto, y a las otras dos distinciones el valor de 2 puntos, todos estos sumandos dan un total de 10 puntos. (ver Tabla 1). Tanto los médicos generales como los cirujanos bien pueden apoyarse

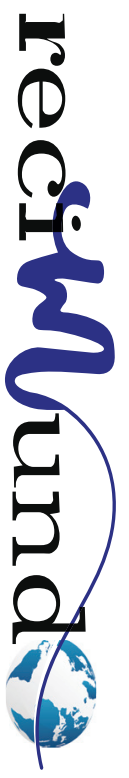


en este sistema a la hora de pronosticar el diagnóstico de apendicitis. (Fernández et al., 2019; Meléndez, Cosio, \& William, 2019)

El resultado que se produce de la sumatoria por la aplicación de este test en particular, directamente se asocia con el riesgo de AA, el cual se puede clasificar en: riesgo bajo (de 0 a 4 puntos), riesgo intermedio (de 5 a 7 puntos) y riesgo alto (de 8 a 10 puntos), siendo los que se ubiquen dentro de éste último grupo los que serán intervenidos quirúrgicamente. (Meléndez, Cosio, \& William, 2019)

Por su parte, Fernández et al. (2019) difiere ligeramente del anterior al considerar que, el riego intermedio culmina con 6 punos, y por ende, el riesgo alto lo asumen a partir de 7 puntos. (pág. 117)

Tabla 1. Escala de Alvarado: Sintoma / Signos / Hallazgos de laboratorio

\begin{tabular}{|l|l|}
\hline \multicolumn{1}{|c|}{ Valor } & \multicolumn{1}{c|}{ Puntaje } \\
\hline M: dolor migratorio & 1 \\
A: anorexia & 1 \\
N: náusea/vómito & 1 \\
T: hiperalgesia en cuadrante inferior derecho & 2 \\
R: signo de Blumberg positivo & 1 \\
E: elevación de temperatura & 1 \\
L: Leucocitosis mayor o igual a 10.000 xmm3 & 2 \\
S: neutrofilia mayor al 75\% & 1 \\
\hline Nota: Adaptado de "Utilidad de la escala de Alvarado en el diagnóstico precoz de apendicitis" \\
Fernández, J. et al. (2019). Universidad, Ciencia y Tecnología (1)1. Pág. 117 \\
\hline
\end{tabular}

Tres de los ochos parámetros antes referidos han sido especialmente destacados como factores claves de diagnóstico, siendo tales: el dolor abdominal, la anorexia y la sensibilidad a la palpación en el cuadrante inferior derecho. (BMJ Best Practice, pág. 9)

El objetivo del presente estudio se centra, mediante el uso de bases de datos y buscadores especializados, en la exploración e identificación de recursos bibliográficos recientes, a fines de analizar, referir e interpretar las ideas, criterios u opiniones de los tratadistas en ellos contenidas respecto al tema de la Escala de Alvarado en la AA. En adelante, se describe la metodología empleada, para proseguir con los resultados obtenidos, en los que se expone como evidencia; definiciones de AA, principales síntomas, etiología y diagnosis fundamental, haciendo una breve ampliación en torno al sistema de Escala de Alvarado, fundamentalmente haciendo referencia de los resul- tados obtenidos en estudios donde dicho método fue aplicado.

\section{Materiales y Métodos}

Conforme al objetivo previamente establecido en esta investigación, se decide adelantar un diseño bibliográfico, bajo una metodología de revisión.

La observación y selección de la bibliografía fue realizada en la última semana de septiembre del corriente por cada uno de los autores del presente estudio, mediante búsquedas en bases de datos (BVS y PubMed), buscadores especializados (SciELO, Medigraphic, Redalyc y Dialnet) y algunos sitios web de revistas científicas vinculadas específicamente al área de la medicina humana y ciencias de la salud, determinadas en base a una búsqueda efectuada en el portal de la Fundación de Ginebra para la Educación y la Investigación Médicas (GF- 
MER).

Este proceso estuvo caracterizado, fundamentalmente, por el empleo palabras clave y operadores lógicos que se construyeron a manera de ecuaciones de búsqueda, estableciendo en concreto: escala de Alvarado + apendicitis aguda; apendicitis aguda + escala de Alvarado; y una configuración de palabras equivalentes en inglés para plataformas en dicho idioma, quedando estructurada la sintaxis: alvarado score + acute appendicitis.

Así, la selección de los contenidos bibliográficos se fue haciendo primordialmente en base a dos paridades; la primera: que respondiera a la mayor coincidencia o correlación entre los títulos referidos en los resultados de las búsquedas y la ecuación o sintaxis utilizada; y segundo: la mayor vinculación que se pudiera evidenciar entre el objeto del tema en cuestión y lo que se diera a entender el contenido del resumen de cada obra; todo ello sin menos cabo de los otros criterios de refinamiento de búsqueda igualmente aplicados, según se hallasen disponibles en cada una de las citadas plataformas de exploración.

En términos generales, esos otros filtros correspondieron a: disponibilidad del contenido (completo); periodo de publicación (2010-2020), idioma (español e inglés); tipo de material bibliográfico (libros digitalizados, e-books, protocolos, consensos, manuales, boletines informativos, ensayos clínicos, tesis de grado, posgrado o doctorado, informes, planes y proyectos, y otras clases de contenidos); además incluyendo guías de práctica clínica, estudios de cohorte y revisiones sistemáticas; que preferiblemente estuviesen producidos, avalados o promovidos por instituciones, entes, organizaciones, sociedades o asociaciones de profesionales en el área de la salud, que fueran de carácter público o privado, nacionales, internacionales o multilaterales.
Se desestimaron: los contenidos repetidos, es decir, no se consideró aquel material que ya se hubiese encontrado y seleccionado previamente mediante algún otro canal de búsqueda utilizado; editoriales o cartas editoriales; anotaciones académicas y otros tipos de materiales bibliográficos escasos de fuentes de sustentación científica o con bajo nivel de evidencia.

Se considera importante aclarar que, en definitiva, tanto ésta metodología como el análisis y la argumentación aquí expuesta fue realizada siempre de manera consensuada.

\section{Resultados}

"La apendicitis aguda es una inflamación aguda del apéndice vermiforme, muy probablemente debida a la obstrucción del lumen del apéndice (mediante fecalito, heces normales, agentes infecciosos o hiperplasia linfoide)" (BMJ Best Practice, 2020, pág. 4)

Fernández et al. (2019) igualmente coinciden en que la $A A$ consiste en el proceso inflamatorio del apéndice cecal, y se asocia con un "alto riesgo de desarrollar íleo, peritonitis, absceso abdominal sino tiene un tratamiento rápido y oportuno". (pág. 116)

\section{Falta Otro concepto}

Las náuseas y los vómitos también están presentes en el $75 \%$ de los pacientes. El estreñimiento absoluto es una característica tardía.

La secuencia de presentación en el 95\% de los pacientes con apendicitis aguda suele comenzar con la anorexia, seguida de dolor abdominal y luego vómitos. Sin embargo, en pacientes embarazadas, las únicas características que se demostró que se asocian significativamente a un diagnóstico de apendicitis son náuseas, vómitos y peritonitis local. (BMJ Best Practice, 2020, pág. 7)

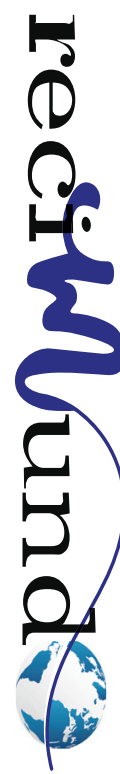




\section{Etiología}

La etiología de la apendicitis aguda es siempre obstructiva. Partiendo del antecedente de que la enfermedad tiene su mayor frecuencia entre los 11 y los 30 años y que dentro de estas edades el pico de incidencia se ubica en los primeros años de la adolescencia, podemos decir que la causa más común de obstrucción apendicular es la hiperplasia del tejido linfoide. Aun cuando se ha demostrado la presencia de bacterias (enterococcus, estreptococcus beta hemolíticus, Escherichia coli), no ha sido posible confirmar si éstas invaden directamente la pared apendicular para causar apendicitis o si llegan por vía hemática o linfática tras el inicio de la enfermedad. Sin embargo, hay un dato importante que se fundamenta en algunos estudios que han demostrado hasta un 5\% de participación directa de Yersinia enterocolitica en la etiopatogenia de la apendicitis aguda. (Rodríguez. 2014 como es citado en (Álvarez, Valdez, \& Ramírez, 2018))

La etiología de la enfermedad involucra la obstrucción del lumen del apéndice vermiforme, lo cual produce acumulación de las secreciones de las células caliciformes, aumento de la presión intraluminal e intramural, lo que resulta en compresión de los vasos de pequeño calibre de la pared del apéndice con la consiguiente estasis linfática e isquemia, que a su vez causa necrosis de la pared. El compromiso de la pared, ocasiona el traspaso de las bacterias desde el lumen intestinal a través de la mucosa hacia la cavidad peritoneal, fenómeno que se denomina translocación bacteriana y produce inflamación transmural del apéndice vermiforme. (Fernández, Sarango, Lema, \& Ibarra, 2019, pág. 116)

El criterio expresado en BMJ Best Practice (2020) en cuanto a la causa de la AA es coincidente con el anterior, pero, en detalle ilustran que:
Los fecalitos (una masa dura de materia fecal), las heces normales o la hiperplasia linfoide son las causas principales de obstrucción. Los datos retrospectivos sobre la apendicectomía sugieren una prevalencia de fecalitos del 14\% al $18 \%$ (entre los pacientes con una indicación clínica/síndrome clínico de apendicitis o los pacientes de apendicectomía de emergencia, respectivamente). En los pacientes sometidos a apendicectomía de urgencia, la prevalencia de fecalitos fue del $39.4 \%$ en la apendicitis perforada, pero solo del $14.6 \%$ en la apendicitis no perforada.

Existe evidencia que sugiere una etiología neuroinmune en algunos casos, pero esto aún se encuentra en proceso de estudio. (pág. 4)

\section{Diagnosis de la AA}

La historia y la exploración física constituyen el enfoque inicial en la evaluación de un paciente con posible apendicitis.

Las herramientas de decisión clínica validadas, como la puntuación de Alvarado, demuestran una gran sensibilidad y son útiles para excluir la apendicitis, pero carecen de especificidad. Se recomienda hacer una ecografía o una resonancia magnética (IRM) del abdomen si la paciente está embarazada. Las mujeres en edad fértil deben someterse a un examen pélvico para descartar otra patología pélvica. (BMJ Best Practice, 2020, pág. 6)

Respecto a las tres medidas más comunes y claves a la hora de diagnosticar la AA, este grupo ha descrito que: el persistente dolor que en principio se ubica a nivel medio del abdomen se desplaza luego al cuadrante inferior derecho en un periodo que puede oscilar entre 1 y 12 horas, siendo más severo si hay tos o movimiento; otro 
de los síntomas más propios es la anorexia, en ausencia de la cual es cuestionable el diagnóstico de la AA; y finalmente esta, la sensibilidad a la palpación en el cuadrante inferior derecho (signo de McBurney), o también los signos de: Blumberg (particularmente, en casos de una presentación de apéndice anterior), Rovsing (dolor en el cuadrante inferior derecho al comprimir el cuadrante inferior izquierdo), psoas (dolor que surge tras recostar al paciente sobre su lado izquierdo y extendiendo lentamente su muslo derecho procurando estirar el músculo psoasilíaco o del obturador (al rotar internamente el muslo derecho flexionado). (pág. 9)

La puntuación de Alvarado utiliza datos de la historia, el examen físico y las pruebas de laboratorio para describir la probabilidad clínica de apendicitis aguda. Aquellos con un puntaje bajo de Alvarado son evaluados para la evaluación de diagnósticos alternativos. En aquellos con un puntaje de Alvarado más alto, se utilizan imágenes y exploración laparoscópica quirúrgica para mejorar la especificidad de la evaluación y minimizar la probabilidad de una laparotomía negativa (Fernández, Sarango, Lema, \& Ibarra, 2019, pág. 116)

En los aportes hallados mediante la presente revisión se evidenció que mayormente consistían en estudios observacionales de corte transversales-descriptivos, con menor frecuencia de los retrospectivos, analíticos y prospectivos, pero que en general su objeto era el evaluar la utilidad diagnóstica, o la sensibilidad y especificidad de la escala de Alvarado (independientemente o en comparación a otras) como herramienta diagnóstica de la $A A$, es por ello que a continuación se hacen referencia de los resultados más relevantes en ellos destacados.

En el tratado de Ospina, Barrera \& Manrique (2011), que tuvo por objeto la evalua- ción de las puntuaciones de la escala de Alvarado en 101 casos clínicos sometidos a cirugía con diagnóstico de AA, llaman la atención, entre los resultados reflejados, los siguientes aspectos:

El tiempo de evolución del cuadro clínico hasta el ingreso registró un rango entre 3 horas y 15 días, con promedio de 44,6 horas para las mujeres y de 40,22 horas para los hombres, aunque la mediana y la moda para esta distribución fueron de 24 horas. Se encontró una diferencia estadísticamente significativa en concordancia de tiempo de evolución mayor de 36 horas y la presentación de necrosis. [...] La media de puntaje registrada con la escala de Alvarado fue de 5,8 (DE=1,86), 5,77 en mujeres y 5,82 en hombres $(p=0,86)$. Por grupos de edad, la media de la escala de Alvarado fue mayor en el grupo de 5 a 14 años $(6,19 ; D E=1,56)$. El análisis de los puntajes de la escala de Alvarado en correlación con el resultado del estudio histopatológico, mostró concordancia diagnóstica de 75,2 \% (...) No se pudieron determinar la sensibilidad y la especificidad, por cuanto no se incluyeron todos los pacientes con cuadro de dolor abdominal, sino los que fueron llevados a cirugía con diagnóstico de apendicitis aguda. [...] En relación con la capacidad discriminante de cada uno de los criterios incluidos en la escala para el diagnóstico de apendicitis aguda con puntaje igual o superior a 7, se encontró que los que resultan más significativos son: el dolor localizado en el cuadrante inferior derecho, la migración del dolor, el signo de Blumberg positivo, la leucocitosis y la neutrofilia $(p<0,01)$; los criterios de náuseas o vómito, temperatura superior a $37,2^{\circ} \mathrm{C}$ y anorexia fueron menos discriminantes ( $p>0,05)$. (pág. 236)

En síntesis, los especialistas concluyen que la escala de Alvarado puede ser una herra-

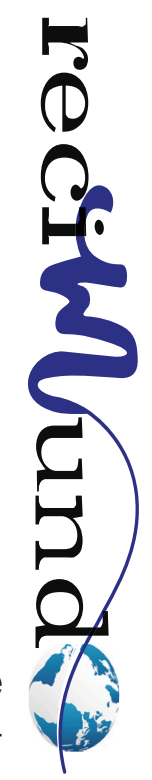


mienta bastante valiosa respecto a la mejorar de la oportunidad y la eficiencia diagnóstica en la AA, muy especialmente en los servicios de atención ambulatoria y de urgencias donde los equipos especializados alto nivel para éste tipo de diagnosis (como las imágenes) no están disponibles.

Entre los resultados de un estudio estadístico mexicano que sí tuvo por objeto la determinación de la sensibilidad y especificidad de la escala de Alvarado en el diagnóstico de apendicitis aguda, mediante la consideración de 384 casos (293 retrospectivos y 91 prospectivos) de pacientes mayores de 14 años, con diagnóstico de probable apendicitis y que fueron operados, fue posible identificar que:

El tiempo de evolución previo a la valoración médica tuvo una media de 40.9 \pm 17.3 horas, rango de 12 a 96 horas, y el tiempo de análisis médico previo a cirugía fue de $5.8 \pm 2.8$ horas, rango de una a 48 horas. [...] Los hallazgos quirúrgicos se compararon con los reportes histopatológicos, obteniendo una concordancia de 96.9. [...] Mediante curvas ROC se determinó que el puntaje más sensible y específico es el punto de corte 5 , siendo $>5$ puntos nuestra referencia. [...] La sensibilidad de los criterios de Alvarado con punto de corte $>5$ fue de $96 \%$ para la población en general, $97 \%$ para hombres y $95 \%$ para las mujeres, con una especificidad de $72 \%$ para la población en general, $61 \%$ para hombres y $83 \%$ para las mujeres. El valor predictivo positivo en la población general fue de 93\%, con 92\% para hombres y $95 \%$ para las mujeres; con un valor predictivo negativo para la población de $82 \%$, hombres con el $81 \%$ y mujeres con el 83\%. [...] Se analizaron los criterios de Alvarado por separado para encontrar su correlación individualmente con el diagnóstico final, encontrando que el criterio más frecuente en los pacientes con sospecha de apendicitis, en el punto de corte $>5$, es el dolor en fosa iliaca derecha con 100\% de pacientes positivos, $72.5 \%$ náusea y vómito, $85 \%$ leucocitosis, 36.5\% dolor migratorio, 54.3\% anorexia, 84.3\% neutrofilia, $48.7 \%$ fiebre, $94.3 \%$ rebote. Sin embargo, al realizar regresión logística binaria se describe el signo de rebote positivo como el más específico para apendicitis aguda. (Hernández \& Domínguez, 2012, págs. 181-1)

Con esos y otros datos llegan a determinar que los criterios de la escala de Alvarado resultan ser útiles para el diagnóstico de AA, pudiendo ser aplicados a cualquier paciente con dolor abdominal agudo sugestivo de apendicitis como prueba al ingreso y para descartar patología apendicular en 5 puntos o menos.

En otros dos estudios de origen cubano (Quesada, Ival, \& González, 2015; López, Fernández, Hernández, \& Pérez, 2016) con diseños investigativos semejantes, básicamente enfocados en la determinación de la utilidad diagnóstica de la escala de Alvarado ante cuadros clínicos sugerentes de AA que presentaron los pacientes atendidos en cada una de las sedes donde se llevó a cabo la aplicación de dicha escala, de manera retrospectiva y prospectiva, respectivamente, logan relacionando los resultados de dicho test con el diagnóstico operatorio y la confirmación histopatológica del diagnóstico, de ambos estudios.

Llama la atención que en los dos estudios los porcentajes de comprobación histológica de la enfermedad son bastante parecidos. De Igual manera, al extrapolar los valores con los que se clasificaron los grupos categorizados como de alto y mediano riesgo, se encuentra que sus resultados fueron coincidentes de manera considerable, ya que fueron mayoría los grupos que se determinaron con puntajes entre 7 y 10 , es decir, que se evidenció como una fortaleza diagnóstica del test. 
Mediante una experiencia local que fue publicada en la misma época por Mena, Muñóz, Barciona, \& Salvador (2015), que también se enfocó en la evaluación de la utilidad de la escala de Alvarado como método diagnóstico para apendicitis aguda, mediante su aplicación durante 10 meses en 268 pacientes mayores de 15 años de edad y menores de 76 años, que presentaron síntomas sugestivos con cualquier tiempo de evolución en el servicio de emergencia del Hospital General "Luis Vernaza", se llegó a determinar que: la edad promedio de presentación se ubicó en los 35 años con una desviación estándar (DS) de \pm 16.42 años (entre hombres y mujeres). Tras la aplicación de la escala de Alvarado a todos los pacientes y se encontró que en el $86.2 \%$ (231 pacientes) de los sujetos se determinó una puntuación $\geq 7$ puntos y en 13.8\% (37 individuos) de los mismos un puntaje $\leq 6$. El diagnóstico de AA pudo confirmarse en 234 pacientes (87.3\%) y se identificaron 34 (12.7\%) de casos de apendicetomías negativas. Se calculó para esta escala una sensibilidad de $96.6 \%$, especificidad de $85.3 \%$, valor predictivo positivo de $97.8 \%$ y valor predictivo negativo de $78.4 \%$.

Finalizan el estudio sosteniendo que sus resultados demuestran la utilidad de la escala de Alvarado como herramienta clínica sencilla con alta sensibilidad, especificidad y exactitud en el diagnóstico de AA. Adicionalmente afirman que la aplicación este método en el triage inicial igualmente resulta ser de gran utilidad, particularmente en los servicios de emergencia, consultorios generales y consultorios rurales que no cuentan con un fácil acceso a estudios de imagen o que presenten una elevada demanda de atención. También destacan las ventajas como procedimiento seguro, rápido,

no invasivo, y de bajo costo que mejora la precisión diagnóstica y consecuentemente reduce el número de apendicectomías negativas; así como también disminuye la tasa de complicaciones atribuidas a un retraso en el diagnóstico de la apendicitis aguda. (págs. 227-8)

Tras la revisión de otros estudios (Siguantay \& Ciraiz, 2016; Concepción et al., 2017; Sandoval, Rodríguez, \& Giangreco, 2017) resulta encontrarse evidencia tendiente a concurrir con los resultados hasta aquí expuestos.

\section{Conclusión}

Sobre la base de las fuentes de datos utilizadas en este estudio es posible deducir que, es indiscutible que el enfoque diagnóstico de la AA se base fundamentalmente en la clínica (historia - exploración física - exámenes de laboratorio) y la imagenología (TC, ecografía, IRM), sin embargo, la Escala de Alvarado, aún posee una prestigiosa aceptación entre los otras escalas (sistemas) de medición validadas (RIPASA, AIR, APACHE II, Ohmann, entre otros) que también se usan al momento de diagnosticar la apendicitis, y ello se debe muy probablemente por la mayor difusión que ésta ha tenido tras haber sido sometida a mayores estudios de validación.

Otro aspecto destacable de esta herramienta diagnóstica es su sensibilidad, bastamente reconocida entre los expertos y múltiples estudios tendiente a ser siempre mayor que la especificidad

En definitiva, la Escala de Alvarado reduce el tiempo de evolución preoperatorio al acelerar la identificación del cuadro clínico, aspecto por demás trascendental ante situaciones de centros de atención de la salud y/o servicios de emergencia con condiciones deficientes o inexistentes de equipamiento imagenológico apropiado para este tipo de patologías. 


\section{Bibliografía}

Álvarez, H., Valdez, F., \& Ramírez, L. (mayo de 2018). Escala de Alvarado como método de diagnóstico en apendicitis aguda. Recimundo, 2(2), 496-524. doi:10.26820/recimundo/2.(2).2018.496-524

BMJ Best Practice. (2020). Apendicitis Aguda. Reino Unido: BMJ Publishing Group Ltd 2020. Recuperado el 25 de septiembre de 2020, de https:// bestpractice.bmj.com/topics/es-es/290/pdf/290/ Apendicitis\%20aguda.pdf

Concepción, I., Fong, M., González, R., Camaño, L., Serrano, T., Quishpe, G., \& Gavilanes, V. (abril-junio de 2017). Escala de Alvarado como herramienta diagnóstica para apendicitis aguda. Enfermería Investiga, Investigación, Vinculación, Docencia y Gestión, 2(2), 68-71. Recuperado el 25 de septiembre de 2020, de enfermeríainvestiga.uta.edu. ec

Fernández, J., Sarango, D., Lema, R., \& Ibarra, S. (Octubre de 2019). Utilidad de la escala de Alvarado en el diagnóstico precoz de apendicitis. Universidad, Ciencia y Tecnología, 1(1), 115-121. Recuperado el 25 de septiembre de 2020, de https:// www.uctunexpo.autanabooks.com/index.php/uct/ article/view/207/294

Hernández, L., \& Domínguez, D. (julio-septimbre de 2012). Sensibilidad y especifi cidad de la escala de Alvarado en apendicitis aguda en el Hospital Regional de Alta Especialidad de Veracruz. Cirujano General, 34(3), 179-184. Recuperado el 25 de septiembre de 2020, de http://www.scielo.org.mx/ pdf/cg/v34n3/v34n3a5.pdf

López, Y., Fernández, A., Hernández, Y., \& Pérez, M. (marzo-abril de 2016). Evaluación de la Escala de Alvarado en el diagnóstico clínico de la apendicitis aguda. Revista Habanera de Ciencias Médicas, 15(2), 213-224. Recuperado el 5 de septiembre de 2020, de http://scielo.sld.cu/scielo.php?script=sci_arttext\&pid=S1729-519X2016000200009

Meléndez, J., Cosio, H., \& William, S. (enero-marzo de 2019). Sensibilidad y especificidad de la Es- cala de Alvarado en el diagnóstico de pacientes atendidos por apendicitis aguda en Hospitales del Cusco. Horizonte Medico, 19(1), 13-18. doi:10.24265/horizmed.2019.v19n1.03

Mena, G., Muñóz, V., Barciona, C., \& Salvador, C. (2015). Utilidad de la escala de Alvarado en el diagnóstico de la apendicitis aguda. Revista Medicina, 19(4), 223-8. doi:10.23878/medicina. v19i4.969

Motta, G., Estrada, D., Romero, C., Santiago, S., García, A., \& Santos, M. (julio-agosto de 2017). Escalas diagnósticas y su utilidad en la evaluación clínica del síndrome doloroso abdominal en el primer escalón de atención médica. Revista de Sanidad Militar, 71(4), 321-331. Recuperado el 25 de septiembre de 2020, de https://www.medigraphic. com/pdfs/sanmil/sm-2017/sm174c.pdf

Ospina, J., Barrera, L., \& Manrique, F. (2011). Utilidad de una escala diagnóstica en casos de apendicitis aguda. Revista colombiana de cirugía, 26(4), 234241. Recuperado el 25 de septiembre de 2020, de https://www.redalyc.org/pdf/3555/355534527009. pdf

Quesada, L. d., Ival, M., \& González, C. (abril-junio de 2015). La escala de Alvarado como recurso clínico para el diagnóstico de la apendicitis aguda. Revista Cubana de Cirugía, 54(2), 121-8. Recuperado el 25 de septiembre de 2020, de http://scielo.sld.cu/ scielo. php?pid=S0034-74932015000200004\&script=sci_arttext\&tIng=en

Sandoval, J., Rodríguez, G., \& Giangreco, M. (abril de 2017). Utilidad de la escala de Alvarado en casos de apendicitis aguda en el Hospital de clínicas Paraguay. Cirugía Paraguaya, 41(1), 18-23. doi:10.18004/sopaci.abril.18-23

Siguantay, M., \& Ciraiz, J. (2016). Implementación de la Escala de Alvarado en Apendicits Aguda. Validación de Prueba. Revista guatemalteca de cirugía, 22, 7 pp. Recuperado el 25 de septiembre de 2020, de http://docs.bvsalud.org/biblioref/2019/09/1016943/1519-31650.pdf 


\section{CITAR ESTE ARTICULO:}

Díaz Castro, I. F., Jonathan Alberto, C. O., Erazo Haro, S. M., \& Moreno Castro, F. I. (2020). Escala de Alvarado en apendicitis aguda. RECIMUNDO, 4(4), 123133. https://doi.org/10.26820/recimundo/4.(4).octubre.2020.123-133 